\title{
Ultrasound Myocardial Elastography and Registered 3D Tagged MRI: Quantitative Strain Comparison
}

Zhen Qian ${ }^{1}$, Wei-Ning Lee ${ }^{2}$, Elisa E. Konofagou ${ }^{2}$, Dimitris N. Metaxas ${ }^{1}$, and Leon $\mathrm{Axel}^{3}$

${ }^{1}$ Center for Computational Biomedicine Imaging and Modeling (CBIM), Rutgers University, New Brunswick, NJ, USA

${ }^{2}$ Department of Biomedical Engineering, Columbia University, New York, NY, USA

${ }^{3}$ Department of Radiology, New York University, New York, NY, USA

\begin{abstract}
Ultrasound Myocardial Elastography (UME) and Tagged Magnetic Resonance Imaging (tMRI) are two imaging modalities that were developed in the recent years to quantitatively estimate the myocardial deformations. Tagged MRI is currently considered as the gold standard for myocardial strain mapping in vivo. However, despite the low SNR nature of ultrasound signals, echocardiography enjoys the widespread availability in the clinic, as well as its low cost and high temporal resolution. Comparing the strain estimation performances of the two techniques has been of great interests to the community. In order to assess the cardiac deformation across different imaging modalities, in this paper, we developed a semi-automatic intensity and gradient based registration framework that rigidly registers the 3D tagged MRIs with the $2 \mathrm{D}$ ultrasound images. Based on the two registered modalities, we conducted spatially and temporally more detailed quantitative strain comparison of the RF-based UME technique and tagged MRI. From the experimental results, we conclude that qualitatively the two modalities share similar overall trends. But error and variations in UME accumulate over time. Quantitatively tMRI is more robust and accurate than UME.
\end{abstract}

\section{Introduction}

Many cardiovascular diseases, such as ischemia and infarction, are associated with the alteration of the global or local contractility of the myocardium. Accurately assessing the detailed myocardial deformation, such as the estimation of the local strain values, could be critical for the early diagnosis of cardiac diseases and dysfunctions. Tagged Magnetic Resonance Imaging (tMRI) and Ultrasound Myocardial Elastography (UME) are two imaging modalities that have been developed in the recent years to quantitatively estimate the myocardial deformations in vivo. The technique of tMRI generates sets of equally spaced parallel tagging planes within the myocardium as temporary material markers at enddiastole through spatial modulation of the magnetization. We have 2 sets of

N. Ayache, S. Ourselin, A. Maeder (Eds.): MICCAI 2007, Part I, LNCS 4791, pp. 800-808, 2007.

(C) Springer-Verlag Berlin Heidelberg 2007 
orthogonal tagging planes for strain assessment. Imaging planes are perpendicular to the tagging planes, so that the tags appear as dark grids and deform with the underlying myocardium during a cardiac cycle. This can yield detailed motion information on the myocardium.

Ultrasound Myocardial Elastography is a radio-frequency (RF) based speckle tracking technique [1. Despite the low SNR nature of ultrasound signals, echocardiography enjoys widespread availability in the clinic, as well as its relatively low cost and high temporal resolution. In UME, the two in-plane orthogonal displacement components (lateral and axial) are estimated using 1D cross-correlation and recorrelation of $\mathrm{RF}$ signals in a $2 \mathrm{D}$ search 2 . Then, the incremental displacements are integrated to obtain a cumulative motion estimation.

Tagged MRI is currently considered the most accurate noninvasive myocardial motion and strain estimator. Several studies have compared the estimates from ultrasound with those from tMRI. Notomi et al. [3], Helle-Valle et al. 4] and Cho et al. 5] have demonstrated that left-ventricular torsion measured from B-mode-based speckle tracking methods is consistent with that from tMRI in short-axis (SA) views. In 6] 2D motion and strain estimates from UME are shown to be highly comparable with those from tMRI. However due to the different characteristics of ultrasonic imaging and tMRI, a main limitation of these comparisons is that the two modalities are not registered in 3D. Thus, the ultrasound and tMR images may not be acquired at the same SA slice with the same orientation. To address this potential discrepancy, in this paper we have developed a semi-automatic intensity and gradient based mutual information registration framework that rigidly registers the $3 \mathrm{D}$ corresponding tagged MRIs with the 2D ultrasonic images. Based on the two registered modalities, we are able to conduct more detailed quantitative strain comparison of the RF-based UME technique and tagged MRI.

\section{Methods}

\subsection{Ultrasound and Tagged MRI Data Acquisition}

Both RF ultrasound and 3D tMRI images were acquired in 2D short-axis (SA) views from two healthy subjects with breath-holding and ECG gating. A clinical echocardiography ultrasound scanner (GE Vivid FiVe, GE Vingmed Ultrasound, Horten, Norway) with a phased array probe (FPA $2.5 \mathrm{MHz} 1 \mathrm{C}$ ) was used to acquire cardiac ultrasound in-phase and quadrature (I/Q) data at the papillary muscle level at a frame rate of $136 \mathrm{fps}$. The I/Q data were upsampled to retrieve the RF signals.

Tagged MR images were obtained from a Siemens Trio 3T MR scanner with $2 \mathrm{D}$ grid tagging. The 3D tagged MR images consists of a stack of 6 equally spaced SA image sets from near the left ventricle (LV) base to the LV apex. The SA orientation of the ultrasound was approximately consistent with that of the tMRI, but was not guaranteed to be the same. Both modalities utilized full ECG gating during the scans so that they can be registered temporally in a heart beat cycle. 


\subsection{Rigid-Body Registration of 2D Elastography with 3D Tagged MRI}

For the purpose of inter-modal comparison, we assume that the overall pattern of the heart shape, size and function of the same subject did not change between acquisition by the different imaging modalities. Previous work on intermodal registration of cardiac ultrasound (US) with MR images has been very limited [7. It has two main difficulties. First, the cardiac left ventricle (LV) in SA view has a circular shape that lacks reliable anatomical landmarks. Second, the US images have very different appearances compared to MR images. In 8 , an approach combining intensity and gradient information was proposed to address the registration of brain US with MR images. In 9] a dynamic cardiac US and MR image registration is achieved by optical tracking of the US probe and fine-tuned by a mutual information registration method.

In our system, since the US probe is not tracked during the imaging process, the semi-automatic registration has to rely on user interactions as well as the image information from the myocardium and the neighboring anatomical structures. The main idea of our registration framework is that we allow the user to freely translate and rotate the US imaging plane w.r.t. the 3D tMRI data and manually find a proper initial registration. Then, a 2D pseudo US image is reconstructed from tMRI, which has an appearance comparable with the $2 \mathrm{D}$ US image, so that a mutual information based method can automatically finetune the initial manual registration by optimizing the translation $\left(t_{x}, t_{y}, t_{z}\right)$ and rotation $\left(\theta_{x}, \theta_{y}, \theta_{z}\right)$ of the imaging plane.

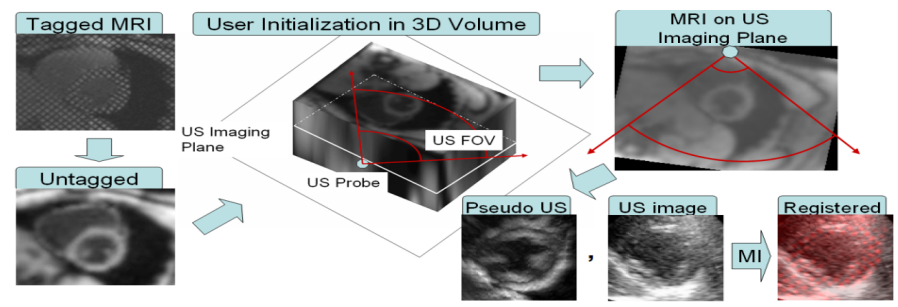

Fig. 1. The flowchart of the rigid-body registration framework. The stack of the untagged images is interpolated using splines to achieve a 3D isotropic volume. The user can freely tune the translation and rotation parameters of the US imaging plane. After manual initialization, a pseudo US image is constructed from the untagged MRI. The position of the simulated US probe gives the US beam direction. Finally an MI based registration procedure is performed to fine-tune the manual initialization.

Both modalities are ECG gated, which easily makes them temporally aligned. First, the tagged MR images at a mid-systolic phase undergo a Gabor filter bankbased tag removal process [10, which essentially enhances the tag-patterned regions. The tag patterns in the blood are flushed out very quickly after the initial tagging modulation, as shown in Fig. 1, this tag removal method enhances the areas of the chest wall, the myocardium and the other surrounding organs, 
and suppresses the areas of the blood pools and lungs. This de-tagging process increases the image's readability. It is helpful for both the user initialization and the following mutual information based registration algorithm.

The characteristic appearance of cardiac US images comes from two main sources. First, the speckle intensity levels vary in different tissues. For instance, we observe that the blood pool area is darker than the myocardium. Second, because of the impedance mismatch effect, US images usually enhance the interfaces between successive tissue layers [8]. We further observe that the angles between the interfaces with the incoming US beam affect the enhancement magnitude. Here we denote vector $\mathbf{b}$ as the direction of the incoming US beam. Therefore we model the transformation $T$ from the tag removed tMRI, $I_{u}$, to the reconstructed image by:

$$
T\left(I_{u}(x, y)\right)=\alpha I_{u}(x, y)+\beta \nabla I_{u}(x, y) \cdot \mathbf{b}(x, y)+\gamma I_{v}(x, y)
$$

The right hand side of the equation is a linear combination of three terms, where the relative weighting parameters $\alpha, \beta$ and $\gamma$ are experimentally determined. The first term is the tag removed image. The second term is an edge detector, which is sensitive to orientation. In order to detect the dark strips between the myocardium and the neighboring liver, the third term $I_{v}$ acts as a valley detector. Suppose $\mathbf{H}(x, y)$ is the Hessian matrix at $I_{u}(x, y)$, and $\lambda_{1}$ and $\lambda_{2}$ are the eigenvalues of $\mathbf{H}$. If $\lambda_{1}<-\left|\lambda_{2}\right|$, then at pixel $(x, y)$ an intensity valley exists, whose width is proportional to $\left|\lambda_{1}\right|$, and its orientation is determined by the eigenvector $\mathbf{v}_{1}$. Hence we model the valley detector as:

$$
I_{v}(x, y)=\left\{\begin{array}{cc}
\lambda_{1}^{2} \mathbf{v}_{1}(x, y) \cdot \mathbf{b}(x, y), \text { if } & \lambda_{1}<-\left|\lambda_{2}\right| \\
0, & \text { otherwise }
\end{array}\right.
$$

The mutual information based optimization of the translation and rotation parameters is found by using gradient decent. Since the tag removed image $I_{u}$ is heavily blurred, the algorithm tends to get stuck in local maxima. Thus a proper manual initialization is necessary. Multiple initializations are also helpful to find the global maximum.

\subsection{Strain Estimation}

Ultrasound Myocardial Elastography. In the UME technique, the two inplane orthogonal displacement components (lateral and axial) were estimated using one-dimensional (1D) cross-correlation and recorrelation of RF signals in a 2D search [2]. The cross-correlation technique employed a 1D matching kernel of $7.7 \mathrm{~mm}$ and $80 \%$ overlap. The reference and comparison frames respectively contained the RF signals before and after deformation. An $8: 1$ linear interpolation scheme between two adjacent original RF signal segments of the comparison frame within the 1D kernel was employed to improve the lateral resolution 2 . The maximal cross-correlated value yielded from the RF signal segment in the comparison frame was considered the best match with the RF signal segment in 
the reference frame. Cosine interpolation was then applied around this maximum of the cross-correlation function for a more refined peak search.

The correction (or, recorrelation) in axial displacement estimation [2, was performed to reduce the decorrelation resulting from axial motion. In UME, recorrelation was implemented by shifting $\mathrm{RF}$ signal segments according to the estimated axial displacement in the comparison frame, prior to the second lateral displacement estimation.

The incremental displacements were integrated to obtain the cumulative displacement that occurred from ED to ES. Appropriate registration for each pixel on two consecutive displacement images was performed to further ensure that the cumulative displacement depicted the motion of the same tissue region.

Strain is defined in terms of the gradient of the displacement. A displacement vector $\mathbf{u}$, is written as $\mathbf{u}=u_{x} \mathbf{e}_{x}+u_{y} \mathbf{e}_{y}$, where $u_{x}$ and $u_{y}$ are lateral and axial displacement components, respectively, and $\mathbf{e}_{x}$ and $\mathbf{e}_{y}$ are the unit vectors in the lateral and axial directions, respectively. The displacement gradient tensor $\mathbf{G}$ in Cartesian coordinates $(x, y)$ is thus defined as $\mathbf{G}=\nabla \mathbf{u}$.

The in-plane Lagrangian finite strain tensor, $\mathbf{E}$, is formulated as in [1]:

$$
\mathbf{E}=\frac{1}{2}\left(\mathbf{G}+\mathbf{G}^{T}+\mathbf{G}^{T} \mathbf{G}\right)
$$

Lateral and axial strains are the diagonal components of $\mathbf{E}$, i.e., $\mathbf{E}_{x x}$ and $\mathbf{E}_{y y}$, respectively. In UME, a least-squares strain estimator (LSQSE) [12 with a kernel of $13.4 \mathrm{~mm}$ in both the lateral and axial directions was used in order to improve the signal-to-noise ratio (SNR) in the strain image (i.e., elastogram) and simultaneously have similar image resolutions between tMRI and UME in order to better facilitate subsequent comparison.

The above mentioned 2D (or, lateral and axial) Lagrangian finite strains are dependent on the orientation of the ultrasound transducer relative to the ventricle. This angle-dependence may complicate the interpretation of the myocardial deformation in the left ventricle. Therefore, radial and circumferential strains are obtained by defining an angle, $\theta$, about the centroid of the left ventricle and by transforming the finite strain tensor $\mathbf{E}$ into a radial-circumferential strain tensor $\dot{\mathbf{E}}$ with a rotation matrix $\mathbf{Q}: \dot{\mathbf{E}}=\mathbf{Q E Q}^{T}[11]$.

Positive and negative radial strains indicate myocardial thickening and thinning, respectively, while myocardial stretching and shortening are represented by positive and negative circumferential strains, respectively.

Tagged MRI. The registered imaging plane may not be the same as one of the tMRI SA slices. Simply interpolating in between the slices might blur the tagging grids and result in inaccurate strain calculations. We observe that the $2 \mathrm{D}$ tagging grids are actually the intersections of two sets of orthogonal tagging sheets and the tMRI imaging planes. If we recover the geometry of the tagging sheets, then by finding their intersections with the registered US imaging plane, we are able to calculate the strain values in the registered imaging plane. Therefore we chose to track the tagging sheets over time [13]. 
First we decompose the grid tagged images into two sets of horizontal and vertical line tagged images by suppressing the component of one direction of the tagging grids via band-stop filtering in the images' Fourier domain. Then the two sets of 3D tagged MR images are filtered with a tunable 3D Gabor filter bank so that the tagging sheets can be enhanced, where the parameters of the 3D Gabor are adaptive to the spacing and orientation of the local tagging sheets. In the tracking step, we impose a set of deformable meshes onto the initial tagging sheets and let them deform according to the enhanced tagging sheets over time. The tracking process is controlled by a dynamic model, and the deformable mesh is smoothed with an internal spring force as well as an inter-mesh spring force.

During tracking, the displacements $\mathbf{u}$ of the intersection points of the two sets of perpendicular tagging sheets and the registered imaging plane are recorded. Then for all the other pixels in the myocardial area, their displacements $\mathbf{u}$ are interpolated by a spline interpolation method.

The strain calculations is the same as that employed in the previous UME section. Lateral, axial, radial and circumferential strains are calculated.

\section{Experimental Results}

In our experiments, active contraction (i.e., systole) was only considered for the assessment of the contractility of cardiac muscle. The strain estimates of these two imaging modalities have qualitatively good agreement. A visual comparison of the strain patterns can be found in Fig. 2, For a normal subject (top panel in Fig. 2), the lateral and axial strains of this clinical data show similar patterns to those of the theoretical framework proposed by Lee et al. 2] . Furthermore, polar strains (bottom panel in Fig. 2) show radial thickening and circumferential shortening except for the anterior and septal walls in the ultrasound images due to the low signal-to-noise (SNR) ratio, which results from the rib and the lung.

As shown in Fig. 4(a), for more spatially localized quantitative analysis, we divide the LV into 6 sectors: the septum 1, the septum 2, the posterior wall, the lateral wall 1, the lateral wall 2, and the anterior wall. According to the quantitative results shown in Fig. 3. both modalities show that the total radial and circumferential strains, from ED to ES, in six different myocardial regions show similar trend of strain value accumulation. In tMRI results, we observe that for intra-subject case, the mean strain values in each sector have similar shapes and slopes, which means the myocardial strain grows evenly and stably over time. Even for inter-subject case, the strain patterns of the two subjects look similar, which shows tMRI strain estimation is quite robust. In addition, the relatively small and stable standard deviations of tMRI strains also show the robustness of tMRI. In UME results, for the case of subject 1, the mean strain values of sectors posterior, lateral 1 and lateral 2, which are opposite to the US probe, are higher than those of the tMRI, while the other 3 sectors, which are near the chest wall and closer to the US probe, have smaller strains. This suggests that UME may be affected by the US beam direction. On the other hand, in general, ultrasound elastographic strain estimates exhibit higher spatial resolution but larger 


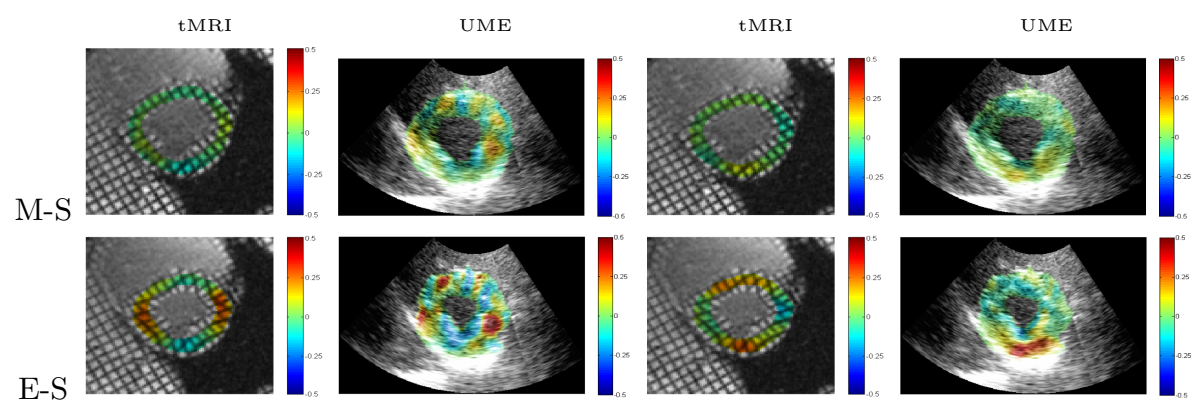

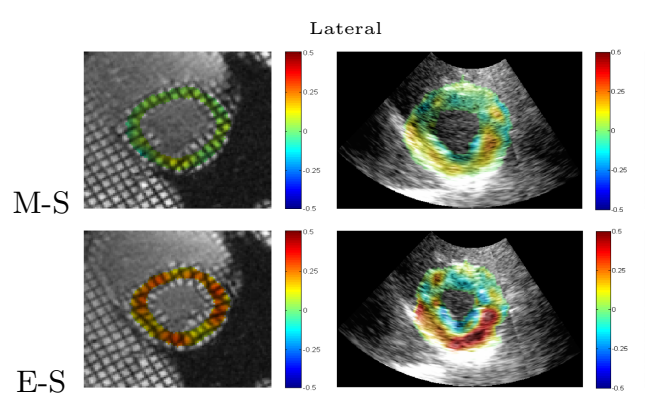

Radial

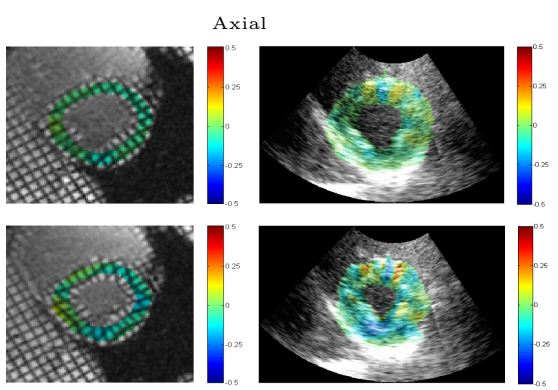

Circumferential

Fig. 2. Strain pattern comparison between tMRI and UME on a healthy subject. Clockwisely starting from the top-left 4 images, lateral, axial, circumferential and radial strains of the left ventricle are displayed. For each strain component, the first row is taken at a mid-systole (M-S) phase, and the second row is taken at end-systole (ES). The pseudo color is displayed on a scale of the strain value from -0.5 to 0.5 .
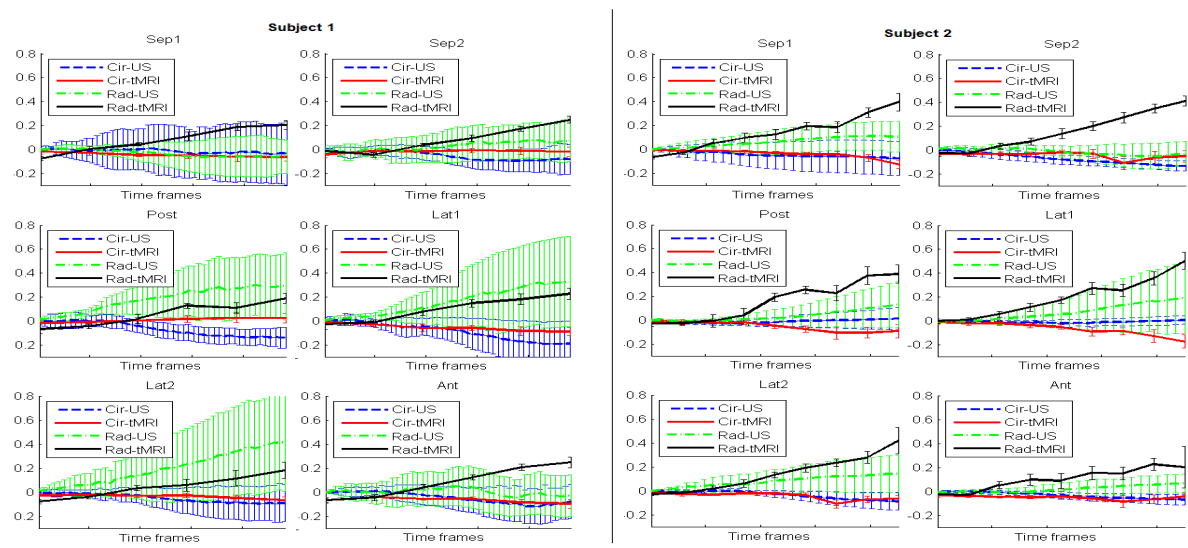

Fig. 3. In each sector of the LV, the mean and standard deviation of the radial and circumferential strain values are calculated from end-diastole to end-systole. We find that the UME results have an overall trend which is similar with that of the tMRI. However the standard deviation of the US results tends to keep growing quickly, while that of the tMRI remains stable. 


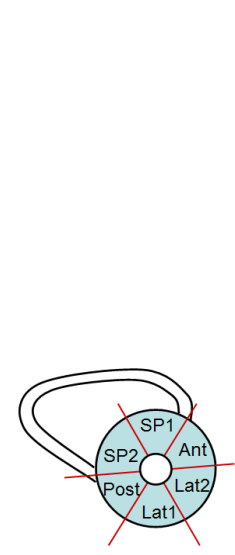

(a)

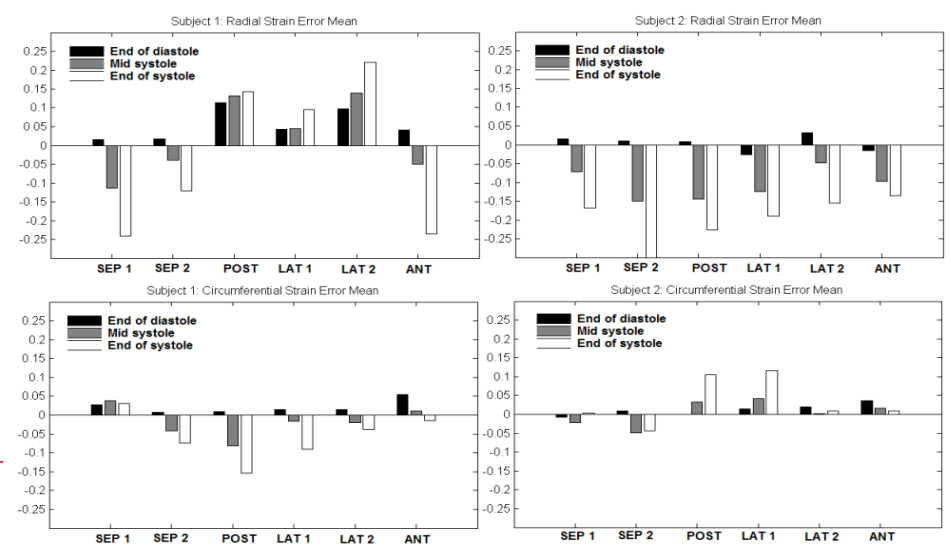

(b)

Fig. 4. (a) illustrates the division of the 6 sectors. (b) is the strain error mean of the UME technique compared with tMRI. We find in UME modality, from ED to ES, the error are also accumulative.

noise. Even though the original pixel resolution in both tagged MR and ultrasonic images is adjusted to the same scale, the overestimation from ultrasound elastography for subject 1 may also owes to its high resolution of estimation. As shown in Fig. 4, the mean strain discrepancies between UME and tMRI increase over from ED to ES. In addition, the ultrasound elastographic estimation errors accumulate during systole can also be depicted from the increasing standard deviations. The circumferential strain estimates depicts the strongest agreement between the two modalities.

\section{Conclusion}

Ultrasound Myocardial Elastography is qualitatively able to assess myocardial motion and deformation with values well comparable to those obtained with tagged MRI in normal subjects. However, for quantitative strain measurement, tMRI will provide much more accurate and robust estimates. Future work will focus on the assessment of the role of the sonographic SNR on the myocardial elastographic strain estimates and study of the tradeoff between spatial resolution and strain accuracy for precise quantification in both normal and acute infarction patients. We will also carry out more UME and tMRI comparisons.

\section{References}

1. Konofagou, E.E., Dhhooge, J., Ophir, J.: Myocardial elastography-a feasibility study in vivo. Ultrasound in Med. \& Bio. 28(4), 475-482 (2002)

2. Lee, W.N., Konofagou, E.E.: Analysis of 3D motion effects in 2D myocardial elastography. In: IEEE-UFFC Symp. Proc. pp. 1217-1220 (2006) 
3. Notomi, Y., et al.: Measurement of ventricular torsion by two-dimensional ultrasound speckle tracking imaging. J. Am. Coll. Cardiol. 45, 2034-2041 (2005)

4. Helle-Valle, T., et al.: New noninvasive method for assessment of left ventricular rotation - speckle tracking echocardiography. Circulation 112, 3149-3156 (2005)

5. Cho, G., et al.: Comparison of two-dimensional speckle and tissue velocity based strain and validation with harmonic phase magnetic resonance imaging. The American Journal of Cardiology 97, 1661-1666 (2006)

6. Lee, W., et al.: Validation of ultrasound myocardial elastography using MR tagging in normal human hearts in vivo. In: ISBI (2007)

7. Makela, T., et al.: A review of cardiac image registration methods. IEEE Trans. Med. Imaging 21(9), 1011-1021 (2002)

8. Roche, A., Pennec, X., Malandain, G., Ayache, N.: Rigid registration of 3D ultrasound with MR images: a new approach combining intensity and gradient information. IEEE Trans. Med. Imaging 20(10), 1038-1049 (2001)

9. Huang, X., et al.: Dynamic 3D ultrasound and MR image registration of the beating heart. In: Duncan, J.S., Gerig, G. (eds.) MICCAI 2005. LNCS, vol. 3750, pp. 171178. Springer, Heidelberg (2005)

10. Manglik, T., et al.: Use of bandpass Gabor filters for enhancing blood-myocardium contrast and filling-in tags in tagged MR images. In: Proc of ISMRM p. 1793 (2004)

11. Lai, W.M.: Introduction to Continuum Mechanics, 3rd edn. ButterworthHeinemann (1993)

12. Kallel, F., Ophir, J.: A least-squares strain estimator for elastography. Ultrasound Imaging 19, 195-208 (1997)

13. Qian, Z., Metaxas, D., Axel, L.: Extraction and tracking of MRI tagging sheets using a 3D Gabor filter bank. In: Proc. of Int'l. Conf. of the Engineering in Medicine and Biology Society (2006) 